\title{
Empagliflozin, an SGLT2 Inhibitor, Reduced the Mortality Rate after Acute Myocardial Infarction with Modification of Cardiac Metabolomes and Antioxidants in Diabetic Rats $\mathbf{\Phi}$
}

\author{
Hiroto Oshima, Takayuki Miki, Atsushi Kuno, Masashi Mizuno, Tatsuya Sato, \\ Masaya Tanno, Toshiyuki Yano, Kei Nakata, Yukishige Kimura, Koki Abe, Wataru Ohwada, \\ and Tetsuji Miura \\ Departments of Cardiovascular, Renal and Metabolic Medicine (H.O., Ta.M., A.K., M.M., T.S., M.T., T.Y., K.N., Y.K., K.A., W.O., \\ Te.M.), Pharmacology (A.K.), and Cellular Physiology and Signal Transduction (T.S.), Sapporo Medical University School of \\ Medicine, Sapporo, Japan
}

Received September 14, 2018; accepted December 10, 2018

\begin{abstract}
The mechanism by which SGLT2 inhibitors reduce cardiac events in diabetic patients remains unclear. Here, we examined the effects of an SGLT2 inhibitor on the acute survival rate after myocardial infarction (MI) in an animal model of type 2 diabetes mellitus (DM) and the possible involvement of modification of cardiac metabolomes and antioxidative proteins. MI was induced in DM Otsuka Long-Evans Tokushima Fatty (OLETF) rats and Long-Evans Tokushima Otsuka (LETO) control rats. Treatment with empagliflozin (10 mg/kg per day, 14 days) before $\mathrm{MI}$ reduced blood glucose and increased blood and myocardial $\beta$-hydroxybutyrate $(\beta \mathrm{OHB})$ levels in OLETF. Survival rate at 48 hours after MI was significantly lower in OLETF rats than in LETO rats (40\% vs. $84 \%)$, and empagliflozin significantly improved the survival rate in OLETF rats
\end{abstract}

to $70 \%$, although the sizes of $\mathrm{Ml}$ were comparable. Patterns of metabolomes and gene expression in the noninfarcted myocardium of OLETF rats were consistent with increased fatty acid oxidation and decreased glucose oxidation. The patterns were modified by empagliflozin, suggesting both increased glucose oxidation and ketone utilization in OLETF rats. Empagliflozin prevented reduction of ATP level in the noninfarcted myocardium after MI and significantly increased myocardial levels of Sirt3 and superoxide dismutase 2 in OLETF rats. Administration of $\beta \mathrm{OHB}$ partially mimicked the effects of empagliflozin in OLETF rats. The results suggest that empagliflozin prevents DM-induced increase in post-Ml mortality, possibly by protective modification of cardiac energy metabolism and antioxidant proteins.

\section{Introduction}

Clinical studies have shown that heart failure (HF), in addition to atherosclerosis-related cardiovascular events, is a major cause of cardiovascular morbidity and mortality in patients with diabetes mellitus (DM) (Shah et al., 2015). DM increases the risk for the development of HF by more than 2-fold (Nichols et al., 2004), and patients with both HF and DM are at particularly high risk for adverse outcomes, regardless of coronary artery disease (Cubbon et al., 2013). Meta-analysis has indicated that intensive glucose control does not prevent

Boehringer Ingelheim did not play any role in the collection, analysis, and interpretation of data or the writing of the manuscript.

This study was supported by a grant from Boehringer Ingelheim, and a Research and Education Grant 2016-2017 from Sapporo Medical University.

Part of this work was presented at the annual meeting of the European Association for the Study of Diabetes, Lisbon, Portugal, September 11-15, 2017.

H.O. and Ta.M. equally contributed to this study.

https://doi.org/10.1124/jpet.118.253666.

S This article has supplemental material available at jpet.aspetjournals.org. cardiac events and rather increased $\mathrm{HF}$ hospitalization compared with standard care (Udell et al., 2015). However, recent clinical trials have demonstrated that treatment with an SGLT2 inhibitor significantly reduced cardiovascular events including HF hospitalization in patients with DM (Zinman et al., 2015; Neal et al., 2017).

How SGLT2 inhibitors prevent HF and death is not completely understood. Treatment of DM patients with SGLT2 inhibitors reduces plasma glucose level and also induces multiple hemodynamic, neurohumoral, and metabolic changes that potentially influence cardiac pathology. Recently, the myocardial fuel hypothesis for cardioprotection afforded by SGLT2 inhibitors has received attention (Ferrannini et al., 2016b; Mudaliar et al., 2016) because SGLT2 inhibitors increase blood $\beta$-hydroxybutyrate $(\beta \mathrm{OHB})$ levels (Ferrannini et al., 2016a; Min et al., 2018). Under physiologic conditions, fatty acid and glucose are the main energy substrates in the heart, where energy sources are switched based on workload and substrate availability.

ABBREVIATIONS: BDH1, 3-hydroxybutyrate dehydrogenase 1; BNP, brain natriuretic peptide; CPT1b, carnitine palmitoyltransferase 1b; DM, diabetes mellitus; HF, heart failure; LETO, Long-Evans Tokushima Otsuka (rats); LV, left ventricular; MI, myocardial infarction; $\beta$ OHB, $\beta$-hydroxybutyrate; OLETF, Otsuka Long-Evans Tokushima Fatty (rats); PPAR, peroxisome proliferator-activated receptor; SCOT, succinyl-CoA: 3-oxoacid CoA transferase; SOD2, superoxide dismutase 2. 
However, HF is associated with decreased glucose oxidation and increased fatty acid oxidation, leading to increased reactive oxygen species in the myocardium (Lopaschuk et al., 2010; Lopaschuk, 2017). Since ketone bodies are more energy efficient than free fatty acids, an increase in circulating ketone bodies is potentially beneficial for failing hearts. Indeed, ketone oxidation is increased in the failing heart (Aubert et al., 2016), and reduction of ketone utilization by cardiomyocyte-specific knockout of succinyl-CoA:3-oxoacid CoA transferase (SCOT), a key enzyme in ketone oxidation, resulted in adverse cardiac remodeling after pressure overload (Schugar et al., 2014). In addition, $\beta$ OHB has been shown to suppress oxidative stress by inhibition of class I histone deacetylases (Shimazu et al., 2013). On the other hand, an association of increase in ketone bodies with adverse events has also been reported. In a study by Mizuno et al. (2017), cardiac uptake of ketone bodies was found to be increased in patients with DM, and the level of ketone uptake was positively correlated with plasma brain natriuretic peptide (BNP) level. Obokata et al. (2017) reported that increased serum $\beta$ OHB level was associated with cardiovascular events and death in patients with renal failure. Thus, whether chronic elevation of circulating ketone bodies induced by an SGLT2 inhibitor is beneficial or detrimental in history of $\mathrm{HF}$ in DM patients remains uncertain.

In the present study, we examined the hypothesis that treatment with an SGLT2 inhibitor reduces mortality after myocardial infarction (MI) by favorable effects on cardiac metabolomes and antioxidant proteins via increased plasma $\beta$ OHB. To test the hypothesis, we used Otsuka Long-Evans Tokushima Fatty (OLETF) rats at ages of 25-30 weeks as a model of DM and we used empagliflozin as an SGLT2 inhibitor. The reason for selection of OLETF rats in this study is 3-fold. First, OLETF rats spontaneously develop DM primarily by hyperphagia due to a lack of cholecystokinin-A receptor in the brain and they show typical phenotype of type 2 DM (obesity, hyperinsulinemia, and hypertriglyceridemia) (Murase et al., 2015; Bi and Moran, 2016). Second, ventricular dysfunction in OLETF rats was characterized in our previous studies (Takada et al., 2012; Kouzu et al., 2015), and shown to be similar to human diabetic cardiomyopathy. Third, we previously found that the mortality rate after MI in OLETF rats was significantly higher, due to lethal $\mathrm{HF}$, than that in Long-Evans Tokushima Otsuka (LETO) rats, the non-DM control rats (Takada et al., 2012; Murase et al., 2015), and thus the beneficial effect of empagliflozin on DM-induced increase in post-MI mortality, if any, should be detectable.

\section{Materials and Methods}

The present study was conducted in strict accordance with the Guide for the Care and Use of Laboratory Animals (https://www.nap. edu/catalog/12910/guide-for-the-care-and-use-of-laboratory-animaleighth) published by National Research Council of the National Academies (2011), Washington, D.C. and was approved by the Animal Use Committee of Sapporo Medical University.

Post-MI Survival Rate Analysis: Protocol 1. The experimental protocol is shown in Fig. 1A. We used male OLETF and LETO rats, at ages of 25-30 weeks. Rats were pretreated with empagliflozin (10 mg/kg per day), an SGLT2 inhibitor, or vehicle (dimethyl sulfoxide and polyethylene glycol; v/v) for 2 weeks. Empagliflozin was kindly provided by Boehringer Ingelheim (Ingelheim am Rhein, Germany). Empagliflozin and the vehicle were administered subcutaneously via
A

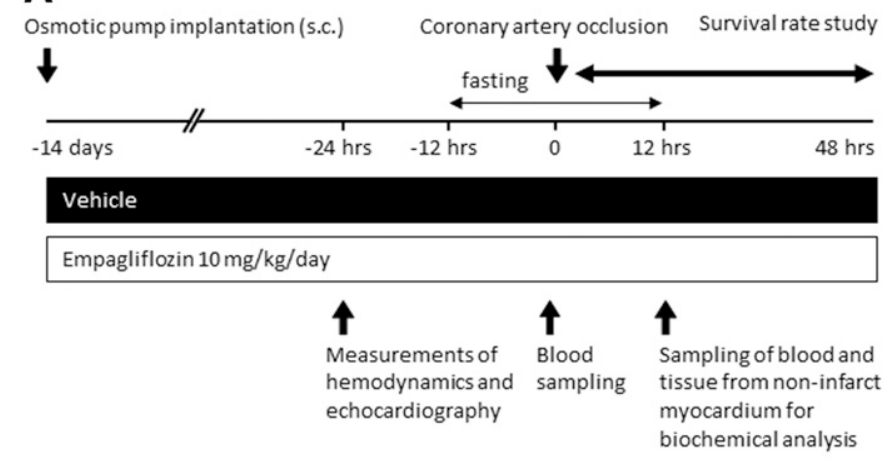

B

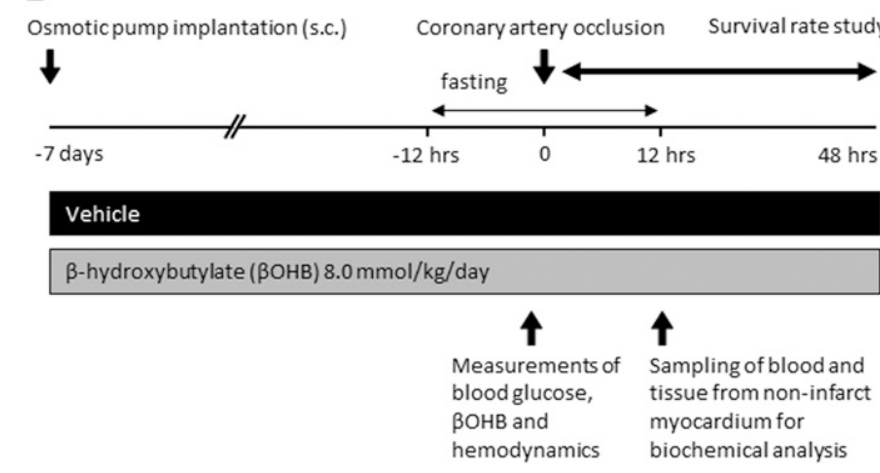

Fig. 1. Experimental protocols. Experimental protocols for survival study and cardiac tissue sampling in empagliflozin experiments (A) and $\beta$-hydroxybutyrate experiments (B).

osmotic minipumps (Alzet, Cupertino, CA) instead of via drinking water containing the agents because the amount of water that rats drink per day is not consistent. One day before induction of MI, systemic blood pressure and pulse rate were measured in a conscious state using a tail-cuff system (BP-98A; Softran, Tokyo, Japan) as in our previous study (Takada et al., 2012). After measurement of hemodynamics, each rat was lightly sedated with $3 \%$ to $4 \%$ isoflurane, and then the chest was shaved and the rat was placed in the supine position. Echocardiography was performed before induction of MI using a $11.5 \mathrm{MHz}$ transducer connected to a Vivid-i Cardiovascular Ultrasound System (GE Healthcare Japan, Tokyo, Japan) as previously reported (Takada et al., 2012; Kouzu et al., 2015; Murase et al., 2015).

After fasting for 12 hours, rats were anesthetized with isoflurane inhalation and then intubated and ventilated with a rodent respirator (model 683; Harvard Apparatus, South Natick, MA). After a small amount of blood was taken from tail vein for measurements of blood glucose and $\beta \mathrm{OHB}$, the heart was exposed via left thoracotomy. Then, a marginal branch of the left coronary artery was permanently ligated using a 5-0 silk thread to induce MI. Since empagliflozin could limit infarct size in the ischemia-reperfusion model (Andreadou et al., 2017), we used a permanent occlusion model of MI to avoid intergroup differences in the infarct size and mechanical stress on the noninfarcted region of the hearts. The surgical wounds were repaired and the rats were returned to their cages. All rats were allowed ad libitum access to water but were restricted from food for 12 hours. Survival rates of rats in four groups (i.e., LETO, empagliflozin-treated LETO, OLETF, and empagliflozin-treated OLETF rats) were determined at 24 and 48 hours after MI. Rats that were alive at 48 hours after MI were euthanized by pentobarbital overdose, and heart tissue was excised. The excised heart was fixed in $10 \%$ formaldehyde and the size of infarction was analyzed by Azan staining and hematoxylin-eosin staining. Blood glucose and $\beta \mathrm{OHB}$ were measured using Glutest-mint (Sanwa Kagaku Kenkyusho, Nagoya, Japan) and Presicion Xceed (Abbot, Chicago, IL), respectively. 
TABLE 1

Metabolic, hemodynamic, and echocardiographic parameters before induction of MI

Values are mean \pm S.E.M.; $N=17-27$.

\begin{tabular}{lcccc}
\hline \multicolumn{1}{c}{ Parameter } & LETO & LETO + EMPA & OLETF & OLETF + EMPA \\
\hline Body weight $(\mathrm{g})$ & $524.7 \pm 6.4$ & $524.0 \pm 7.7$ & $645.0 \pm 7.8^{*}$ & $644.4 \pm 6.2^{\dagger}$ \\
Blood glucose $(\mathrm{mg} / \mathrm{dl})$ & $124.2 \pm 2.7$ & $112.9 \pm 3.6$ & $170.7 \pm 6.9^{*}$ & $116.4 \pm 6.4^{\ddagger}$ \\
$\beta$ OHB $(\mathrm{mmol} / \mathrm{l})$ & $0.77 \pm 0.03$ & $1.37 \pm 0.07^{*}$ & $0.62 \pm 0.2^{*}$ & $1.17 \pm 0.08^{\dagger, \neq}$ \\
Heart rate $(\mathrm{bpm})$ & $342.7 \pm 6.4$ & $330.2 \pm 4.9$ & $324.4 \pm 5.6$ & $326.8 \pm 5.3$ \\
Mean BP $(\mathrm{mmHg})$ & $105.8 \pm 2.6$ & $100.6 \pm 2.9$ & $122.7 \pm 3.3^{*}$ & $120.3 \pm 2.1^{\dagger}$ \\
FS $(\%)$ & $44.2 \pm 1.1$ & $47.4 \pm 1.1$ & $45.4 \pm 1.0$ & $44.5 \pm 1.0$ \\
IVST $(\mathrm{mm})$ & $1.72 \pm 0.04$ & $1.71 \pm 0.04$ & $1.73 \pm 0.03$ & $1.77 \pm 0.03$ \\
PWT $(\mathrm{mm})$ & $1.82 \pm 0.07$ & $1.80 \pm 0.05$ & $1.83 \pm 0.04$ & $1.82 \pm 0.08$ \\
LVEDD $(\mathrm{mm})$ & $8.36 \pm 0.11$ & $8.58 \pm 0.18$ & $8.86 \pm 0.09^{*}$ & $8.85 \pm 0.09$ \\
\hline
\end{tabular}

BP, blood pressure; bpm, beats per minute; EMPA, empagliflozin; FS, fractional shortening; IVST, interventricular septal thickness; LVEDD, left ventricular end-diastolic dimension; PWT, posterior wall thickness.

$* P<0.05$ vs. LETO; ${ }^{\dagger} P<0.05$ vs. LETO + EMPA; ${ }^{\dagger} P<0.05$ vs. OLETF.

Post-MI Survival Rate Analysis: Protocol 2. To examine the effects of exogenous $\beta \mathrm{OHB}$ on mortality, we subcutaneously administered $\beta \mathrm{OHB}(8.0 \mathrm{mmol} / \mathrm{kg}$ per day) or vehicle to OLETF rats for 7 days before MI. After 12 hours of fasting, MI was induced as in protocol 1 (Fig. 1B).

Tissue Sampling Protocol. We previously reported that the mortality rate at 24-48 hours after MI was high (approximately $70 \%$ ) in OLETF rats at ages of 25-30 weeks (Takada et al., 2012; Murase et al., 2015). Thus, myocardial tissue sampling for biochemical analyses was performed at 12 hours after MI (Fig. 1). Since empagliflozin did not affect the survival rate of LETO rats (see Results), we used noninfarcted heart tissue samples from MI-induced rats (i.e., LETO, OLETF, and empagliflozin-treated OLETF rats) to analyze changes associated with the empagliflozin-induced protection in this series of experiments. At 12 hours after MI, hearts were quickly excised and immediately soaked in ice-cold saline, and myocardium in the noninfarcted region was excised and frozen in liquid nitrogen within 30 seconds. The samples were stored at $-80^{\circ} \mathrm{C}$ until use for biochemical analyses as in our previous studies (Takada et al., 2012; Murase et al., 2015). The hearts treated with vehicle or $\beta \mathrm{OHB}$ in OLETF rats (protocol 2) were also sampled at 12 hours after MI.

Cellular Signaling Analysis. Frozen tissue samples were homogenized in ice-cold buffer (CelLyticTM MT Cell Lysis Reagent) including protease and phosphatase inhibitor cocktails (Nacalai Tesque, Inc., Kyoto, Japan). The homogenate was centrifuged at $15,000 \mathrm{~g}$ for 15 minutes at $4^{\circ} \mathrm{C}$ to obtain the supernatant. Equal amounts of protein were analyzed by immunoblot assays using specific antibodies (Supplemental Table 1). Intensities of individual bands were quantified by using Image $J$ software (National Institutes of Health, Bethesda, MD). mRNA quantification was performed as in our previous studies (Takada et al., 2012; Kouzu et al., 2015; Murase et al., 2015). Taqman gene expression assays and primer sequences are shown in Supplemental Table 1. ATP content in the noninfarcted myocardium was measured using an ATP colorimetric/fluorometric assay kit (BioVision Inc., Milpitas, CA). The formation of malondialdehyde and 4-hydroxynonenal, an indicator of lipid peroxidation, was examined using a Lipid Peroxidation Microplate Assay Kit (Oxford Biomedical Research, Oxford, MI).

Metabolome Analyses. In this experiment, analysis was performed by Basic Scan and C-Scope (Human Metabolome Technologies, Yamagata, Japan), according to the recommended protocol (Makinoshima et al., 2014). Concentrations of extracted metabolites were measured by capillary electrophoresis time-of-flight mass spectrometry and capillary electrophoresis tandem mass spectrometry as described previously (Makinoshima et al., 2014).

Statistical Analyses. Data are presented as the mean \pm S.E.M. Differences between treatment groups after MI were assessed by oneway analysis of variance. The Student-Newman-Keuls post hoc test was used for multiple comparisons when analysis of variance indicated significant differences. Survival rates after MI were compared by Kaplan-Meier curves and log-rank statistics. For all tests, $P<0.05$ was considered statistically significant.

\section{Results}

Metabolic and Hemodynamic Profiles. Data for physiologic profiles before induction of MI in the survival experiment are shown in Table 1. OLETF rats had larger body weight and higher fasting blood glucose than those of LETO rats, and treatment with empagliflozin reduced blood glucose level without affecting body weight in OLETF rats. Empagliflozin significantly increased $\beta$ OHB level both in LETO and OLETF rats. Mean blood pressure, but not heart rate, in a conscious state was higher by $10-20 \mathrm{~mm} \mathrm{Hg}$ in OLETF rats than in LETO rats, and empagliflozin did not significantly change these parameters.

Echocardiography revealed that there were no differences in left ventricular (LV) fractional shortening and LV wall thickness between LETO and OLETF rats, although LV dimension was larger in OLETF rats than in LETO rats before MI. Treatment with empagliflozin did not alter these echocardiographic parameters in either LETO or OLETF rats (Table 1). We tried to assess LV function after MI, but echocardiographic images of sufficient quality for analyses could not be obtained because of the surgical wound and remaining intrathoracic air.

Data for metabolic and hemodynamic parameters before MI in the sampling experiment were similar to those in the survival experiment (data not shown). At 12 hours after MI (i.e., 24 hours of fasting), the levels of blood glucose and serum insulin were higher in OLETF rats than in LETO rats (Table 2). Treatment with empagliflozin significantly reduced blood glucose level but not serum insulin level in OLETF rats. The level of $\beta \mathrm{OHB}$ was slightly increased by continuation of fasting and by induction of MI in LETO and OLETF rats, and it was markedly increased in empagliflozin-treated OLETF rats. Levels of total cholesterol and triglyceride were higher in OLETF rats than in LETO rats, and the change in the lipids was attenuated by empagliflozin. Acidosis was not induced by empagliflozin even after MI and 24 hours of fasting.

Infusion of exogenous $\beta \mathrm{OHB}$ did not change body weight and blood glucose, but significantly increased the level of blood $\beta$ OHB by approximately $30 \%$ in OLETF rats (Table 3 ). Exogenous $\beta \mathrm{OHB}$ slightly decreased blood pressure without affecting heart rate before MI in OLETF rats. 
TABLE 2

Metabolic parameters at 12 hours after MI (before tissue sampling)

Values are mean \pm S.E.M.; pH was measured by blood gas analysis; $N=11-16$.

\begin{tabular}{lccc}
\hline \multicolumn{1}{c}{ Parameter } & LETO & OLETF & OLETF + EMPA \\
\hline Blood glucose (mg/dl) & $135.9 \pm 7.3$ & $197.4 \pm 9.1^{*}$ & $114.9 \pm 7.1^{\dagger}$ \\
Serum insulin $(\mathrm{ng} / \mathrm{ml})$ & $0.94 \pm 0.04$ & $1.27 \pm 0.09^{*}$ & $1.23 \pm 0.08^{*}$ \\
$\beta$ OHB (mmol/l) & $1.08 \pm 0.08$ & $1.33 \pm 0.14$ & $4.20 \pm 0.50^{*} \dagger$ \\
Total cholesterol (mg/dl) & $97.4 \pm 5.1$ & $116.3 \pm 9.2^{*}$ & $87.1 \pm 4.6^{\dagger}$ \\
Triglyceride $(\mathrm{mg} / \mathrm{dl})$ & $21.6 \pm 5.7$ & $180.3 \pm 25.6^{*}$ & $118.2 \pm 18.3^{*}$, \\
pH & $7.40 \pm 0.02$ & $7.44 \pm 0.01$ & $7.38 \pm 0.02$ \\
\hline
\end{tabular}

EMPA, empagliflozin

${ }^{*} P<0.05$ vs. LETO; ${ }^{\dagger} P<0.05$ vs. OLETF.

Survival Rate after MI. The survival rate during a period of 48 hours after MI in LETO rats was $84.2 \%$, and pretreatment with empagliflozin for 2 weeks before MI did not affect the survival rate in LETO rats $(88.2 \%)$ (Fig. 2A). As in our previous studies (Takada et al., 2012; Murase et al., 2015), the survival rate was significantly lower in OLETF rats (40.0\%) than in LETO rats. Pretreatment of OLETF rats with empagliflozin significantly increased the survival rate $(70.4 \%)$ (Fig. 2A). Autopsies of rats that died within 48 hours after MI revealed lung congestion without cardiac rupture. Infarct sizes 48 hours after permanent coronary occlusion were $31 \%-37 \%$ of the left ventricle and were comparable among the treatment groups (Fig. $2 \mathrm{C}$ ). In $\beta$ OHB-treated OLETF rats, the survival rate was higher by $45 \%$ than that in vehicletreated OLETF rats $(76.2 \%$ vs. $52.6 \%$ ) (Fig. $2 \mathrm{~B}$ ), although the difference did not reach statistical significance.

Myocardial Levels of $\beta O H B$, ATP, and BNP mRNA. $\beta$ OHB levels in the noninfarcted myocardium after MI were similar in LETO and OLETF rats $(190 \pm 33$ and $184 \pm 31 \mathrm{nmol} / \mathrm{g}$ of wet tissue) (Supplemental Table 2). Treatment with empagliflozin significantly increased the $\beta$ OHB level in OLETF rats $(1719 \pm 670 \mathrm{nmol} / \mathrm{g}$ of wet tissue $)$. The myocardial ATP level after MI was significantly lower in OLETF rats than in LETO rats $(3550 \pm 502$ vs. $5693 \pm$ $435 \mathrm{nmol} / \mathrm{g}$ of wet tissue) (Fig. 2D). In contrast, the ATP level was preserved in empagliflozin-treated OLETF rats after MI $(5719 \pm 789 \mathrm{nmol} / \mathrm{g}$ of wet tissue). The preserved myocardial ATP level in empagliflozin-treated OLETF rats was similarly observed in metabolome analyses (as detailed subsequently and in Supplemental Table 2), although the difference did not reach statistical significance because of the small number of samples in this analysis $(n=4)$. In a different set of experiments, we found that the myocardial ATP level was not reduced after $\mathrm{MI}$ in $\beta$ OHB-treated OLETF rats

\section{TABLE 3}

Metabolic and hemodynamic parameters before MI in ketone infusion study

Values are mean \pm S.E.M.; $N=13-15$.

\begin{tabular}{lcc}
\hline \multirow{2}{*}{ Parameter } & \multicolumn{2}{c}{ OLETF } \\
\cline { 2 - 3 } & Vehicle & $\beta$ OHB \\
\hline Body weight $(\mathrm{g})$ & $666.8 \pm 6.1$ & $663.1 \pm 9.9$ \\
Blood glucose $(\mathrm{mg} / \mathrm{dl})$ & $163.4 \pm 7.4$ & $143.5 \pm 9.4$ \\
$\beta$ OHB $(\mathrm{mmol} / \mathrm{l})$ & $0.62 \pm 0.03$ & $0.82 \pm 0.04^{*}$ \\
Heart rate $(\mathrm{bpm})$ & $318.4 \pm 6.7$ & $331.3 \pm 8.8$ \\
Mean blood pressure $(\mathrm{mmHg})$ & $117.8 \pm 2.3$ & $109.2 \pm 2.4^{*}$ \\
\hline
\end{tabular}

bpm, beats per minute.

$* P<0.05$ vs. vehicle.
( $5216 \pm 1023 \mathrm{nmol} / \mathrm{g}$ of wet tissue, $n=6$ ). The BNP mRNA level after MI in OLETF rats was slightly reduced by empagliflozin treatment, but there was no statistical difference in BNP mRNA levels among the three groups (Fig. 2E).

Expression of Genes that Regulate Glucose Metabolism, $\beta$-Oxidation, and Ketone Oxidation. The mRNA levels of glucose transporter types 1 and 4 in the myocardium were not different between LETO and OLETF rats (Fig. 3, A and B). Empagliflozin did not affect these mRNA levels in OLETF rats. SGLT1 mRNA was detectable at similarly low levels in LETO and OLETF rats (data not shown) and SGLT2 mRNA was not detected in the myocardium. The mRNA levels of peroxisome proliferator-activated receptor (PPAR)- $\alpha$ and CD36 were higher in OLETF rats than in LETO rats, and empagliflozin treatment did not change these levels in OLETF rats (Fig. 3, C and D). On the other hand, the increased levels of both mRNA and protein of carnitine palmitoyltransferase $1 b(C P T 1 b)$ in OLETF rats were attenuated by empagliflozin (Fig. 3, E and J). Expression of FoxO3a mRNA was increased in OLETF rats regardless of empagliflozin treatment (Fig. 3F). Protein levels of 3-hydroxybutyrate dehydrogenase 1 (BDH1) and SCOT were not different among LETO, OLETF, and empagliflozin-treated OLETF rats (Fig. 3, G and H). MCT1 protein, a transporter of lactate, pyruvate, and ketone bodies, was also similarly expressed in the three groups (Fig. 3I).

Metabolome Analyses. Major metabolites relevant to energy metabolism in the noninfarcted region of the heart are shown in Fig. 4. The levels of glucose 6-phosphate and fructose 1,6-diphosphate were similar in OLETF and LETO rats. The ratio of fructose 1,6-diphosphate to fructose 6-phosphate was significantly reduced in OLETF rats without MI (Kouzu et al., 2015), indicating suppressed phosphofructokinase; however, such a change in the ratio of fructose 1,6-diphosphate to fructose 6-phosphate was not observed in OLETF rats with MI in this series of experiments. On the other hand, the lactate level and lactate/pyruvate ratio were increased in OLETF rats, suggesting impaired glucose oxidation by reduced flux of pyruvate into the trichloroacetic acid cycle with diversion to lactate. Acetyl CoA levels were similar in LETO and OLETF rats, and the maintenance of acetyl CoA level in OLETF rats may be explained by increased $\beta$-oxidation since the expression levels of PPAR- $\alpha$, CD36, and CPT1b and protein level of CPT1b were increased in the myocardium (Fig. 3, C-E and J). Ketone bodies are unlikely to compensate for the diversion of pyruvate to lactate in OLETF rats since myocardial $\beta \mathrm{OHB}$ levels were similar in LETO and OLETF rats. Pentose phosphate pathway intermediates and ratios of NADPH to 


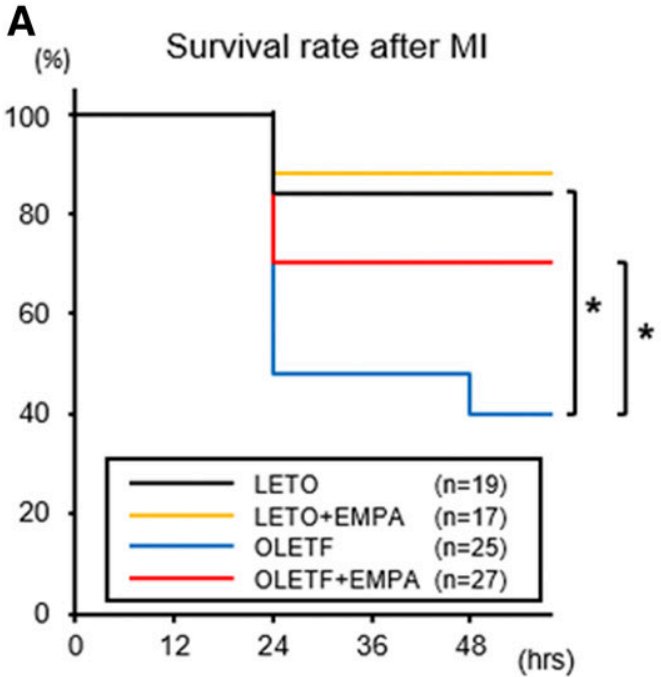

B
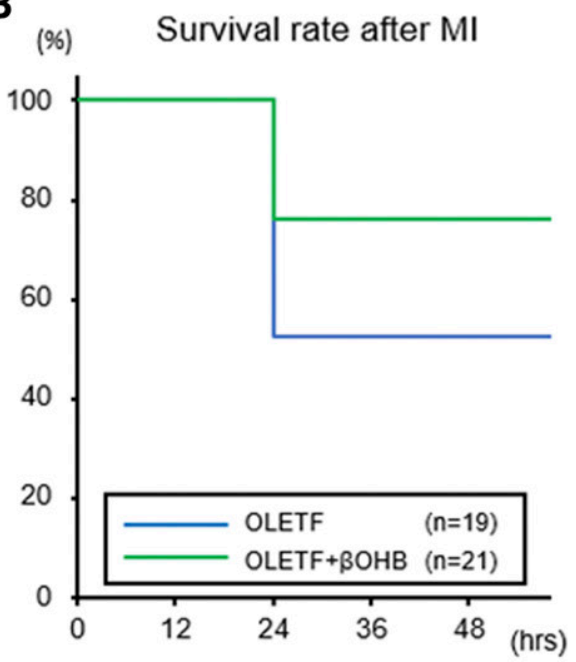

C
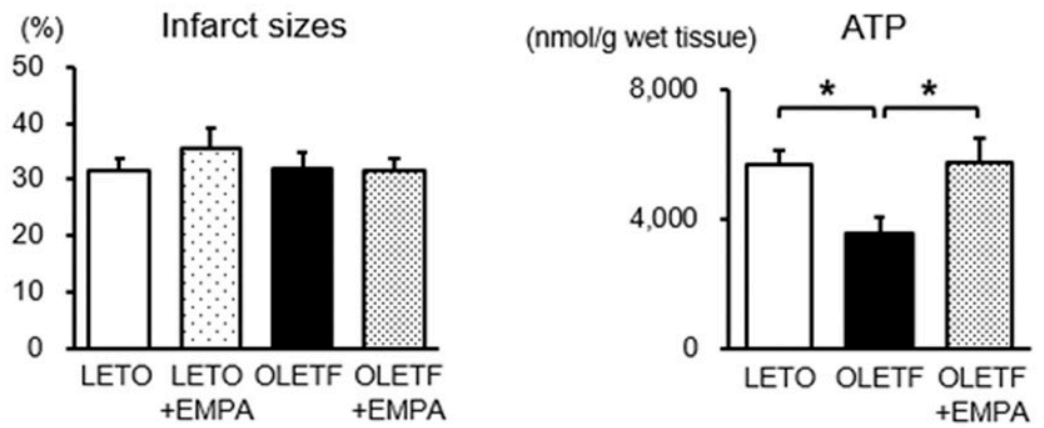

Fig. 2. Effects of empagliflozin on survival rate, myocardial ATP, and BNP after MI. (A) Kaplan-Meier survival analysis of LETO rats treated with a vehicle (LETO), LETO rats treated with empagliflozin (LETO + EMPA), OLETF rats treated with a vehicle (OLETF), and OLETF rats treated with empagliflozin (OLETF + EMPA). $* P<0.05$. (B) KaplanMeier survival analysis of OLETF rats treated with a vehicle (OLETF) and OLETF rats treated with $\beta$-hydroxybutyrate $(\mathrm{OLETF}+\beta \mathrm{OHB})$. (C) Infarct size measured at 48 hours after MI. (D) ATP level in the noninfarcted myocardium sampled 12 hours after MI. $N=6$ to 7 in each group. $* P<0.05$. (E) BNP mRNA levels normalized to $\beta$-actin in the noninfarcted myocardium after MI. $N=8$ in each group. ${ }^{*} P<0.05$. a.u., arbitrary unit.

E

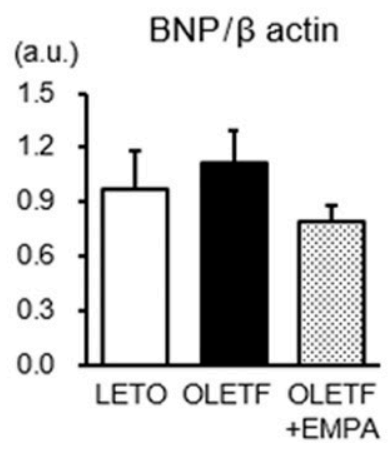

$\mathrm{NADP}^{+}$and reduced glutathione to oxidized glutathione were not different in LETO and OLETF rats (Fig. 4; Supplemental Table 2). Levels of most tricarboxylic acid cycle intermediates (fumarate, malate, and citrate, but not succinate) were slightly decreased in OLETF rats. The decline in ATP in OLETF rats was associated with increased levels of ADP and AMP, and total adenylate levels were therefore comparable in LETO and OLETF rats.

In empagliflozin-treated OLETF rats, the levels of 3-phosphoglyceric acid and phosphoenolpyruvic acid were similar to those in vehicle-treated OLETF rats, but the pyruvate level was markedly reduced. The change in pyruvate level was accompanied by reductions in lactate level, lactate/pyruvate ratio, and alanine level, suggesting that flux of pyruvate into the tricarboxylic acid cycle was increased by empagliflozin (Fig. 4; Supplemental Table 2). It is unlikely that $\beta$-oxidation of fatty acids contributed to the maintenance of acetyl CoA production in OLETF rats because mRNA and protein levels of CPT1b were reduced (Fig. 3, E and J) and lipid droplets in the cardiomyocytes were rather increased by empagliflozin (Mizuno et al., 2018). Therefore, increased myocardial $\beta \mathrm{OHB}$ level (Fig. 4) might have contributed to acetyl CoA production, although lack of data for proteins and metabolites in the pathway from $\beta \mathrm{OHB}$ to acetyl CoA does not allow us to draw the conclusion regarding this point.

Antioxidant Proteins and Reactive Oxygen Species Production. The level of malondialdehyde plus 4-hydroxynonenal 
A

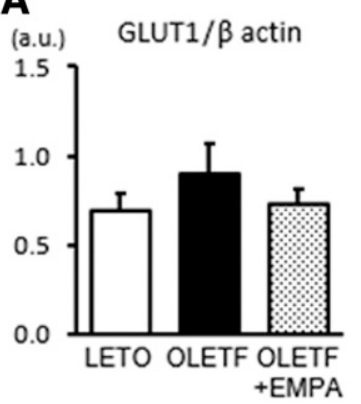

D $\quad \mathrm{CD} 36 / \beta$ actin

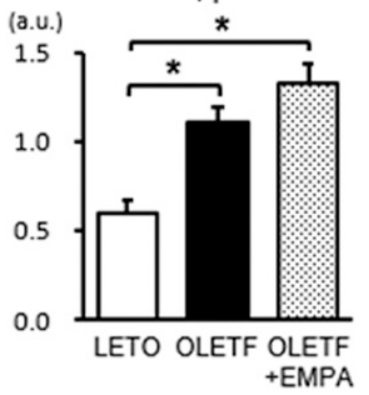

B

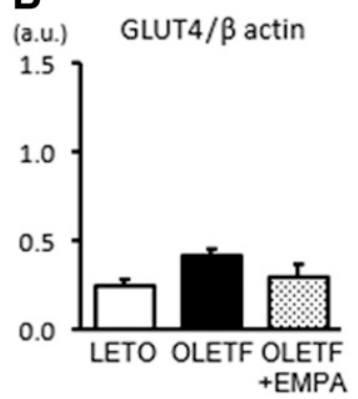

E $\quad \mathrm{CPT} 1 \mathrm{~b} / \beta$ actin

(a.u.)

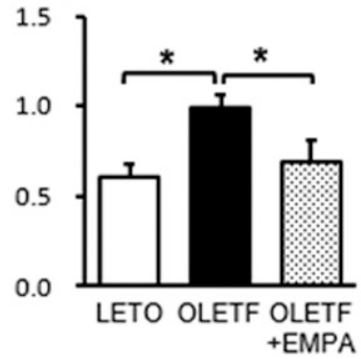

C

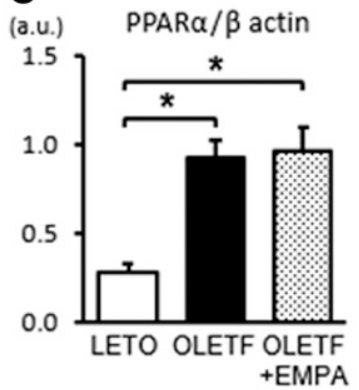

F FoxO3a/ $\beta$ actin

(a.u.)

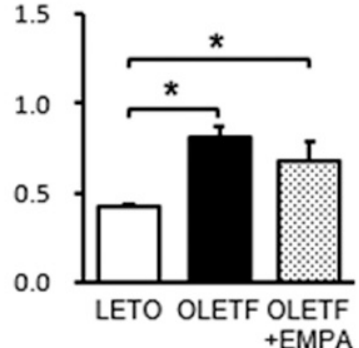

G

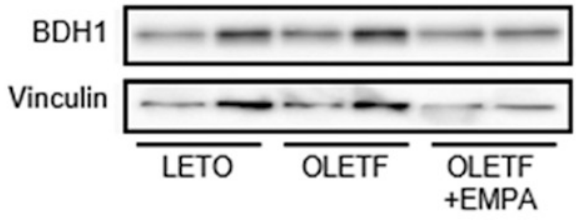

H

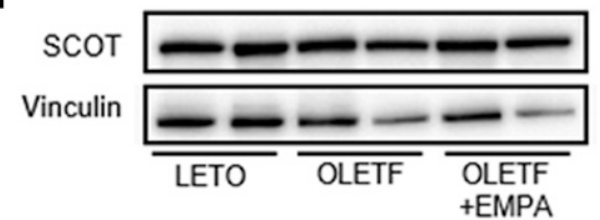

I

MCT1

Vinculin
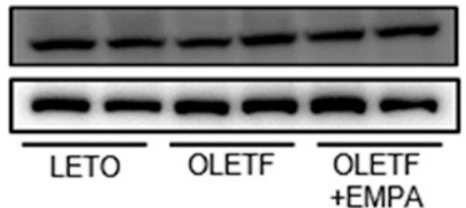

$\mathbf{J}$

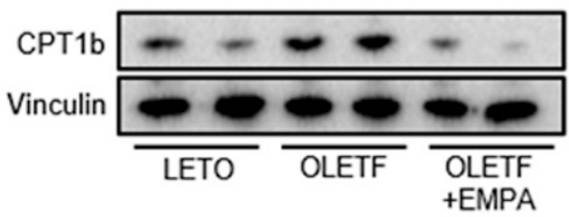

\section{(a.u.)}

\section{0}

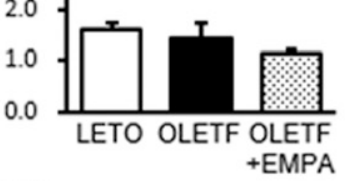

(a.u.)
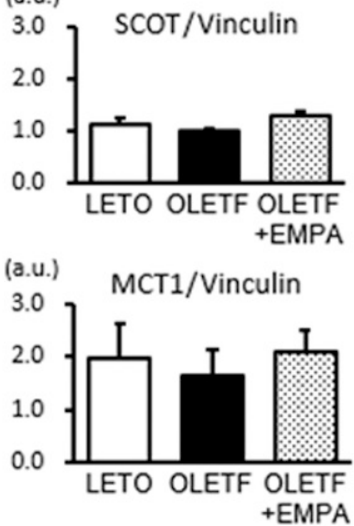

(a.u.) $\mathrm{CPT} 1 \mathrm{~b} /$ Vinculin

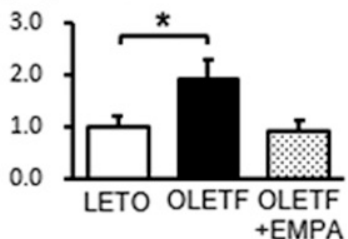

Fig. 3. Expressions of molecules regulating energy substrate metabolism. mRNA levels of glucose transporter type 1 (GLUT1) (A), glucose transporter type 4 (GLUT4) (B), PPAR $\alpha$ (C), CD36 (D), CPT1b (E), and FoxO3a (F) in the myocardium. ${ }^{*} P<0.05 . N=4-9$ in each group. Representative immunoblot (left) and summary data (right) of BDH1 (G), SCOT (H), MCT1 (I), and CPT1b (J) protein levels normalized by vinculin in the noninfarcted myocardium after MI in LETO, OLETF, and empagliflozintreated OLETF rats (OLETF + EMPA). $N=6-8$ in each group. a.u.= arbitrary unit. in the noninfarcted myocardium after MI was significantly higher in OLETF rats than in LETO rats, and this increase in OLETF rats was attenuated by treatment with empagliflozin (Fig. 5A). Protein levels of superoxide dismutase 2 (SOD2) were similar in OLETF and LETO rats, but catalase level was lower in OLETF rats (Fig. 5, B and C). Empagliflozin treatment increased the SOD2 level and partially restored the catalase level in OLETF rats. The level of acetylated histone H3 was modestly increased by empagliflozin, although the difference did not reach statistical significance (Fig. 5D). The protein level of Sirt3, a mitochondrial sirtuin, in OLETF rats was similar to that in LETO rats but was significantly increased by treatment with empagliflozin (Fig. 5E). The effects of empagliflozin on malondialdehyde plus 4-hydroxynonenal level, antioxidant proteins, 

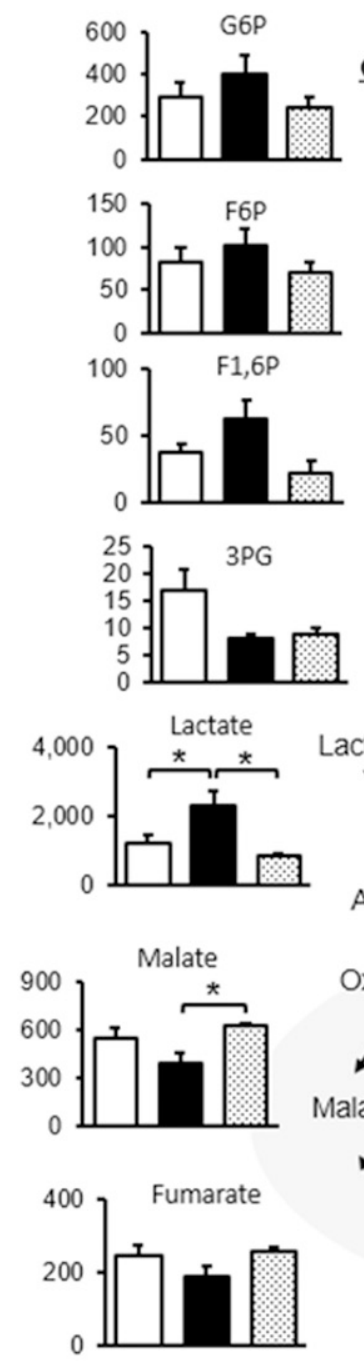

Adenylate

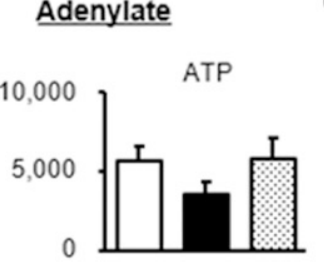

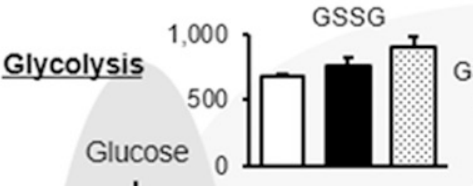
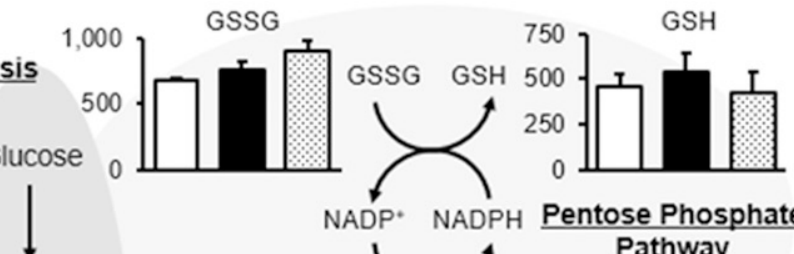

ADP. NADPH

Pentose Phosphate

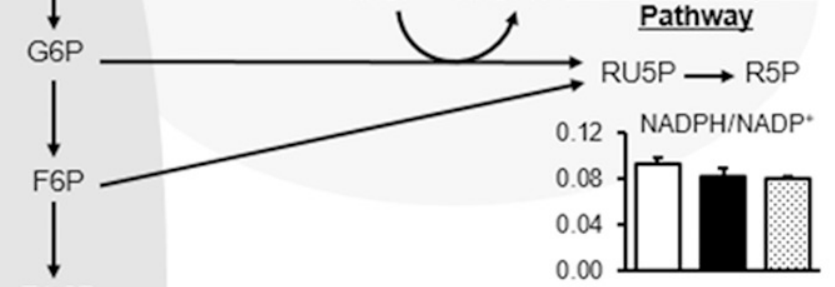

$$
\mathrm{F} 1,6 \mathrm{P}
$$<smiles>[Mg][TeH]</smiles>
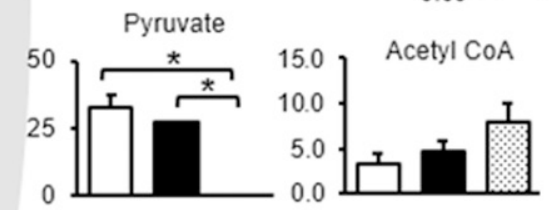

Ketone and Lipid oxidation
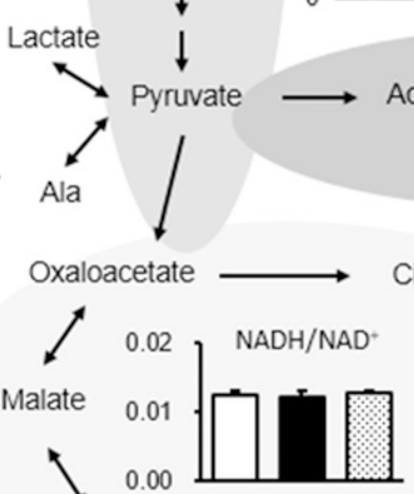

Fumarate

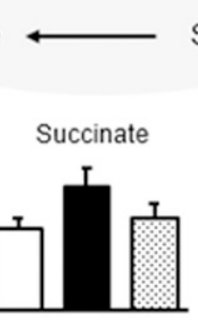

Succinate
Fig. 4. Summary of changes in metabolomics profile. Metabolites of the energy production pathway [glycolysis, ketone and lipid oxidation, and tricarboxylic acid (TCA) cycle] and pentose phosphate pathway. Concentrations of all metabolites are expressed as nanomoles per gram of wet tissue. $* P<0.05 . N=4$ in each group. AcCoA, acetyl CoA; AAcCoA, acetoacetyl CoA; F1,6P, fructose 1,6-diphosphate; F6P, fructose 6-phosphate; G6P, glucose 6-phosphate; GSH, reduced glutathione; GSSG, oxidized glutathione; 3PG, 3-phosphoglyceric acid; R5P, ribose-5phosphate; Ru5P, ribulose-5-phosphate. and Sirt3 were mimicked by exogenous administration of $\beta \mathrm{OHB}$ in OLETF rats (Fig. 6).

\section{Discussion}

In the present study, treatment with empagliflozin for 2 weeks significantly improved the survival rate of OLETF rats after acute $\mathrm{MI}$ (Fig. 2A). We previously demonstrated that increased mortality during the acute phase of MI in OLETF rats is due to progressive $\mathrm{HF}$ but not lethal arrhythmia (Takada et al., 2012). Congestion of the lung but not cardiac rupture after MI was detected by post-mortem examinations in rats that died before 48 hours after MI. Furthermore, BNP expression at 12 hours after MI in OLETF rats was slightly suppressed by empagliflozin (Fig. 2E). Therefore, suppression of $\mathrm{HF}$ is the most likely explanation for reduction in mortality after MI in OLETF rats by empagliflozin.

Unlike its effect in DM patients (Zinman et al., 2015; Neal et al., 2017), a beneficial effect of empagliflozin on post-MI mortality was observed without a significant change in body weight, blood pressure, or heart rate in OLETF (Tables 1 and 2). Thus, we could exclude hemodynamic changes from mechanisms of alterations in myocardial metabolism by empagliflozin. Changes in patterns of myocardial metabolites by empagliflozin in OLETF rats are consistent with the notion that empagliflozin restored glucose oxidation, increased 
A

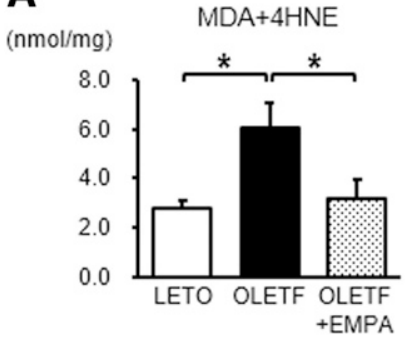

B

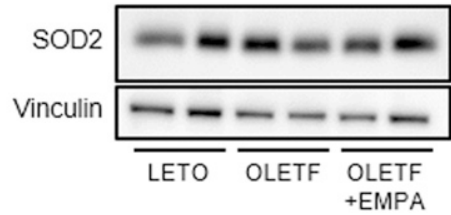

C

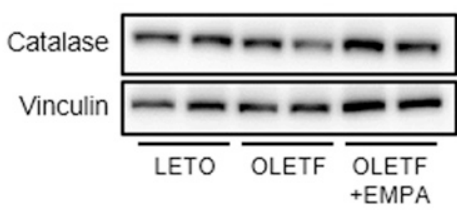

D

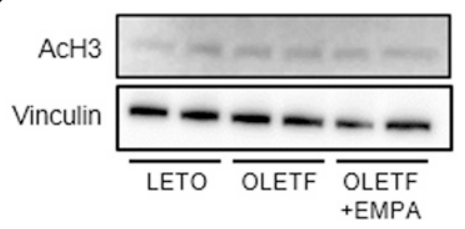

$\mathbf{E}$

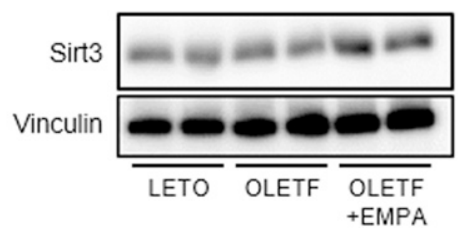

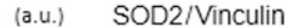
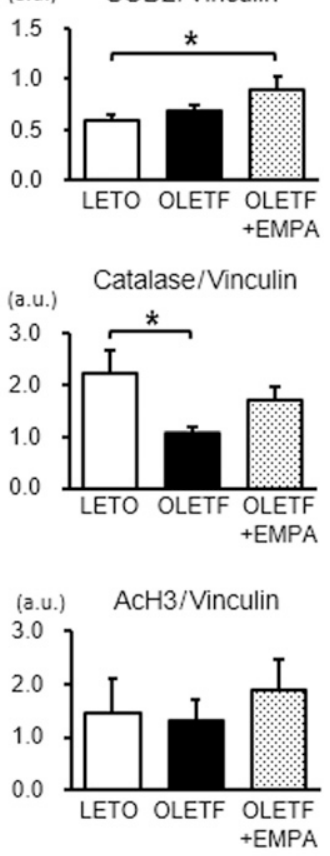

Sirt3/Vinculin

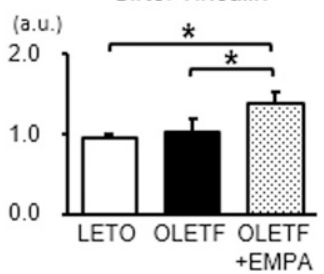

Fig. 5. Effects of empagliflozin on lipid peroxidation and antioxidant proteins. (A) Malondialdehyde (MDA) plus 4-hydroxynonenal (4HNE) in the noninfarcted myocardium after MI in LETO, OLETF, and empagliflozin-treated OLETF rats (EMPA). $N=6-8$ in each group. Representative immunoblot (left) and summary data (right) of SOD2 (B), catalase (C), acetylated histone H3 (D), and Sirt3 (E) protein levels normalized by vinculin in the noninfarcted myocardium after MI in LETO, OLETF, and empagliflozin-treated OLETF rats (OLETF + EMPA). $* P<0.05 . N=6-8$ in each group. AcH3, acetylated histone H3; Sirt3, $\mathrm{NAD}^{+}$-dependent deacetylase sirtuin-3; SOD, superoxide dismutase; a.u., arbitrary unit.

ketone oxidation, and decreased fatty acid oxidation, leading to maintenance of the ATP level in the myocardium of OLETF rats (Figs. 2D and 4). In contrast, Verma et al. (2018) recently reported that empagliflozin increased the rate of glucose and fatty acid oxidation without change in the ketone oxidation rate in $\mathrm{db} / \mathrm{db}$ mice. We do not have a clear explanation for the difference in empagliflozin-induced changes in cardiac metabolism between OLETF rats and db/db mice, but the presence of MI-induced HF in this study might be involved. Nevertheless, both the study by Verma et al. (2018) and the present study indicate that empagliflozin affords beneficial effect on

A

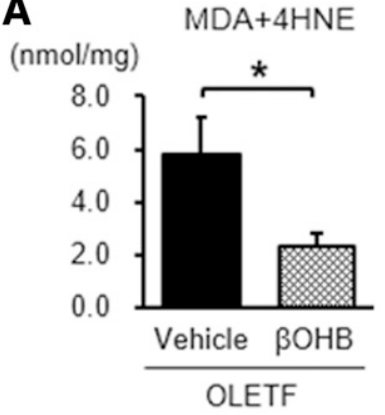

B
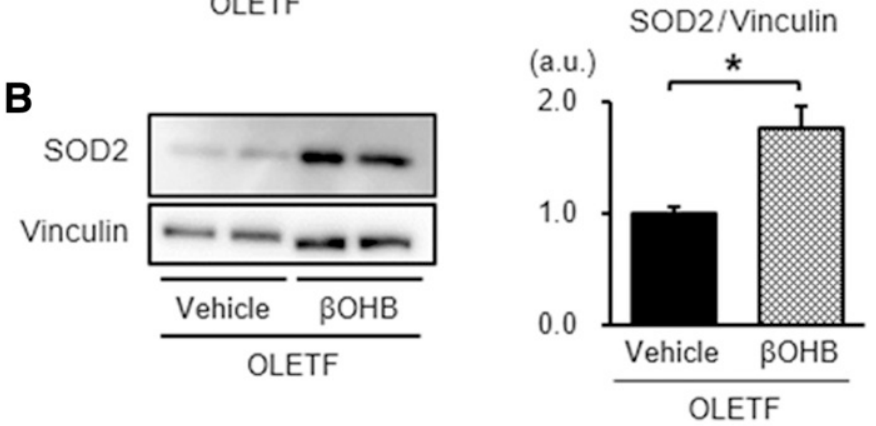

C

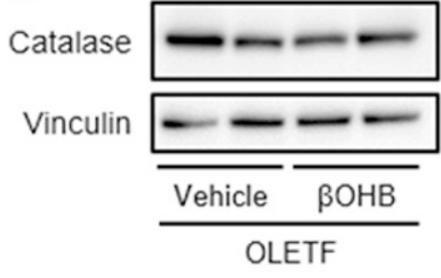

(a.u.) Catalase/Vinculin

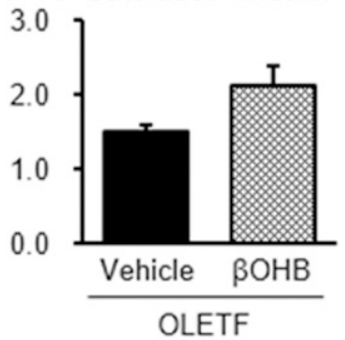

D
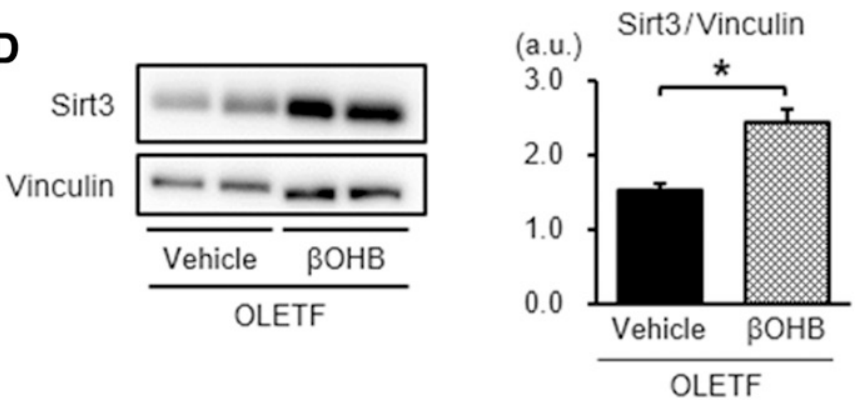

Fig. 6. Effects of $\beta \mathrm{OHB}$ on lipid peroxidation and antioxidant proteins. (A) Malondialdehyde (MDA) plus 4-hydroxynonenal (4HNE) in the noninfarcted myocardium after MI in OLETF and $\beta$-hydroxybutyratetreated OLETF rats $(\beta \mathrm{OHB}) . * P<0.05 . N=6-8$ in each group. Representative immunoblot (left) and summary data (right) of SOD2 (B), catalase (C), and Sirt3 (D) protein levels normalized by vinculin in the noninfarcted myocardium after MI in OLETF and $\beta$-hydroxybutyratetreated OLETF rats $(\beta \mathrm{OHB}) . * P<0.05 . N=6-8$ in each group. Sirt3, $\mathrm{NAD}^{+}$-dependent deacetylase sirtuin-3; SOD, superoxide dismutase.

myocardial ATP level. In addition to the changes in energy substrate metabolism, suppression of oxidative stress was another important change in empagliflozin-treated OLETF rats, since major roles of oxidative stress in development of $\mathrm{HF}$ have been shown in animal models of $\mathrm{HF}$ and patients with HF (Münzel et al., 2015; Ayoub et al., 2017). We found that empagliflozin increased expression of antioxidant stress proteins and suppressed biomarkers of oxidant stress in the 


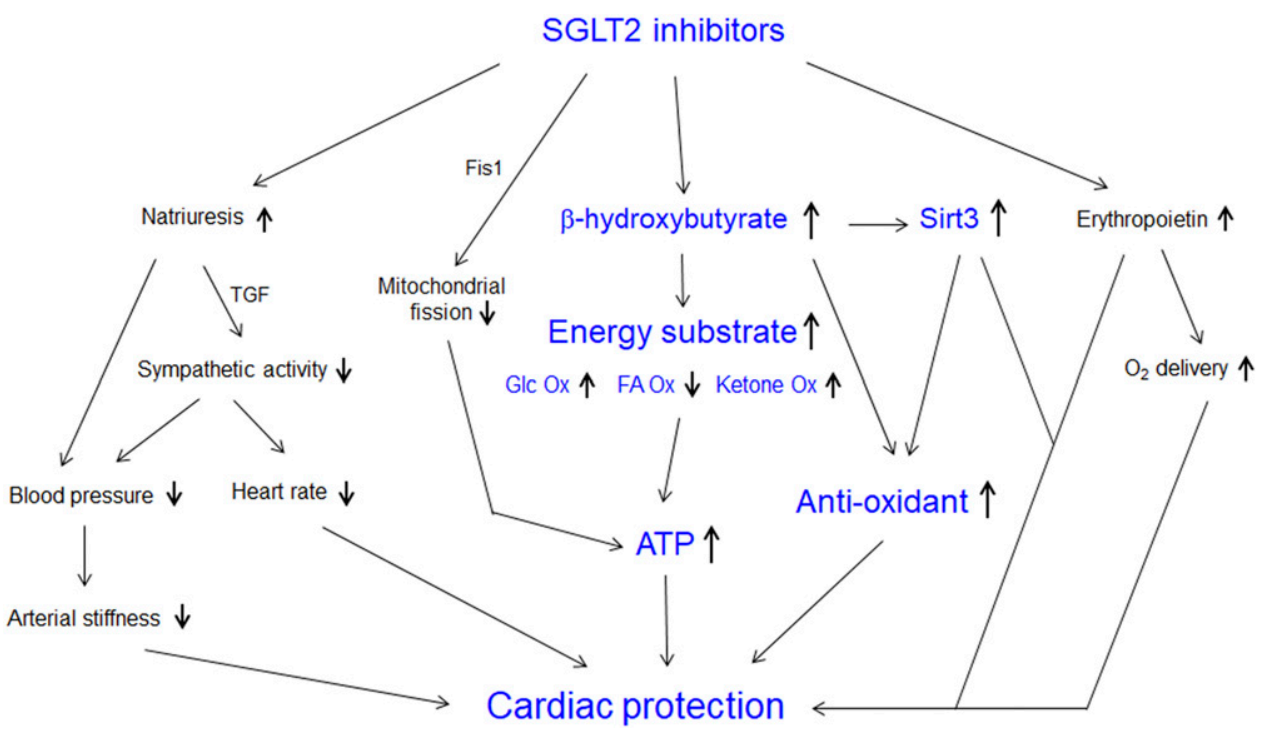

Fig. 7. Scheme of possible mechanisms by which SGLT2 inhibitors protect the myocardium from lethal heart failure after MI. The mechanisms suggested by the results of the present study are highlighted in blue. FA Ox, fatty acid oxidation; Fis 1, fission 1; Glc Ox, glucose oxidation; TGF, tubuloglomerular feedback.

myocardium after MI in OLETF rats (Fig. 5, A-C). Exogenous infusion of $\beta \mathrm{OHB}$ partially mimicked the protective effects of empagliflozin on mortality, ATP level, and oxidative stress after MI in OLETF rats (Figs. 2B and 6). The findings support the notion that improved function of the noninfarcted myocardium after MI by suppression of myocardial oxidant stress underlay improvement of post-MI survival rate in empagliflozin-treated OLETF rats.

It has been shown that a failing heart switches the main energy sources from fatty acid to glucose metabolism, which is more efficient fuel (Lopaschuk et al., 2010; Lopaschuk, 2017). However, when $\mathrm{HF}$ reduces glucose oxidation by reduction of pyruvate dehydrogenase activity, it leads to a mismatch between glycolysis and glucose oxidation (Diakos et al., 2016). On the other hand, ketone oxidation increases to compensate for the decrease in acetyl CoA production in the failing heart (Aubert et al., 2016; Bedi et al., 2016). Recently, it has been reported that cardiac-specific overexpression of BDH1 was protective in mice, while knockout of SCOT was detrimental to HF induced by pressure overload (Schugar et al., 2014; Uchihashi et al., 2017). These results support the notion that promotion of ketone oxidation improves the energy metabolism in failing hearts. In the present study, myocardial $\beta O H B$ level was not different in LETO and OLETF rats and there was no change in the protein level of MCT1, BDH1, or SCOT after MI in OLETF rats (Figs. 3, G-I, and 4). Thus, the present model of MI-induced HF did not have upregulated ketone oxidation in the myocardium in contrast to HF models in earlier studies (Schugar et al., 2014; Aubert et al., 2016; Bedi et al., 2016; Diakos et al., 2016; Uchihashi et al., 2017), possibly because the duration of cardiac overload ( 12 hours vs. 4-8 weeks), severity of HF (post-MI vs. aortic banding model), and/or presence of DM were different. Nevertheless, treatment with empagliflozin increased blood and myocardial $\beta \mathrm{OHB}$ levels by approximately 2 - and 10 -fold, respectively, and the increased tissue level of $\beta \mathrm{OHB}$ was accompanied by preservation of ATP level after MI. These findings suggested the possibility that increased delivery of ketone bodies to the heart with ventricular dysfunction is beneficial in terms of energy metabolism even if the expression of MCT1, BDH1, and SCOT is not upregulated. However, metabolic adaptation of the heart may change during much longer treatment with empagliflozin, since it has been reported that a chronic ketogenic state in mice decreased myocardial SCOT expression and prevented inhibition of fatty acid oxidation (Wentz et al., 2010).

Oxidative stress plays a major role in myocardial dysfunction in HF and complications of DM (Münzel et al., 2015; Ayoub et al., 2017; Niemann et al., 2017), and suppression of reactive oxygen species improved $\mathrm{LV}$ function after MI (Kinugawa et al., 2000). Recent studies have shown that expression of antioxidant enzymes, including SOD2 and catalase, are increased by the class I histone deacetylase inhibitory action of $\beta \mathrm{OHB}$ via upregulation of FoxO3a (Shimazu et al., 2013; Nagao et al., 2016). In the present study, the myocardial SOD2 level was unchanged, but the catalase level was lower in OLETF rats than in LETO rats (Fig. 5, B and C). Empagliflozin increased the SOD2 level and restored the catalase level in OLETF rats, although it had no significant effect on FoxO3a mRNA or acetylated histone 3 protein levels. On the other hand, the Sirt3 protein level was significantly increased in empagliflozin-treated OLETF rats, and one of the functions of Sirt3 is upregulation of SOD2 and catalase by deacetylation of FoxO3a (Tseng et al., 2014). Interestingly, administration of exogenous ketones mimicked upregulation of Sirt3 and antioxidant protein expression, being consistent with a report that infusion of ketones increased Sirt3 protein expression in brain tissues (Yin et al., 2015). How infusion of $\beta$ OHB upregulated Sirt3 expression in OLETF rats is unclear. However, activation of nuclear respiratory factor 2 has been reported to mediate upregulation of Sirt3 expression by ketones in noncardiac cells (Satterstrom et al., 2015; Buler et al., 2016; Izuta et al., 2018). Mechanisms by which $\beta \mathrm{OHB}$ activates nuclear respiratory factor 2 and other regulatory factors of Sirt3 expression in the myocardium remain to be further investigated.

Although activators of PPARs have been shown not only to normalize blood glucose level but also to ameliorate decreased glucose oxidation and increased fatty acid oxidation in diabetic hearts, the agents failed to improve cardiac 
performance (Aasum et al., 2002, 2005; Carley et al., 2004). Treatment with insulin in isolated perfused hearts of $\mathrm{db} / \mathrm{db}$ mice rather reduced ATP production by reduction of palmitate oxidation (Verma et al., 2018). As effects being distinct from the effects of PPAR activators and insulin, empagliflozin increased ketone utilization in OLETF rats, in addition to restoring glucose oxidation and decreasing fatty acid oxidation, and upregulated antioxidant proteins (Figs. 4 and 5). Taken together, increased utilization of ketones and suppression of myocardial oxidant stress may play larger roles than improved glucose and fatty acid metabolism in empagliflozininduced protection of diabetic hearts from acute mortality after MI.

There are limitations in the present study. First, although metabolome analysis provided abundant data for metabolites, this method does not allow direct measurement of the flux in metabolic pathways. Thus, the assessment of cardiac energy metabolism made in this study is interpretive. Isotope labeling of metabolites in an isolated perfused heart preparation is a more rigorous method to determine metabolic fluxes, but it is difficult for an ex vivo perfusion study to mimic the effects of SGLT2 inhibitors under in situ conditions because of lack of organ interactions, including ketone body supply from the liver and kidney to the heart. Second, a role of $\beta \mathrm{OHB}$ in empagliflozin-induced cardioprotection has not been clarified. Infusion of exogenous $\beta$ OHB into OLETF rats mimicked the effect of empagliflozin on Sirt3 and antioxidant proteins, but its effect on myocardial metabolomes was not analyzed. Since it was technically difficult to elevate the blood $\beta \mathrm{OHB}$ level by its infusion to the level of $\beta \mathrm{OHB}$ in empagliflozin-treated OLETF rats, we did not attempt to rigorously compare the effects of exogenous $\beta \mathrm{OHB}$ and empagliflozin on myocardial metabolomes. Third, whether the cardioprotective effect of empagliflozin is dose dependent and/or is glycemic control dependent remained unclear. However, infusion of $\beta \mathrm{OHB}$ partly mimicked the effects of empagliflozin on antioxidant protein expression and mortality after MI, without significant reduction in blood glucose, in OLETF rats. The findings are consistent with results of clinical trials arguing against the presence of a close relationship between the level of glycemic control and the effects of SGLT2 inhibitors on cardiovascular events (Zinman et al., 2015; Neal et al., 2017).

In conclusion, treatment with empagliflozin significantly attenuated DM-induced increase in acute mortality after MI in a model of type 2 DM. The protective effects of an SGLT2 inhibitor were independent of hemodynamic changes and hematopoiesis but were associated with improved energy metabolism and upregulation of antioxidative proteins in the remote region of post-MI hearts (Fig. 7). How the changes in cardiac metabolism and antioxidant defense play roles in the protection afforded by empagliflozin against the detrimental effect of DM on post-MI mortality warrants further investigation.

\section{Authorship Contributions}

Participated in research design: Miki, Miura.

Conducted experiments: Oshima, Miki, Kuno, Mizuno, Sato, Nakata, Kimura, Abe, Ohwada.

Performed data analysis: Oshima, Miki, Kuno, Sato, Tanno, Yano.

Wrote or contributed to the writing of the manuscript: Oshima, Miki, Kuno, Tanno, Miura.

\section{References}

Aasum E, Belke DD, Severson DL, Riemersma RA, Cooper M, Andreassen M, and Larsen TS (2002) Cardiac function and metabolism in type 2 diabetic mice after treatment with BM 17.0744, a novel PPAR- $\alpha$ activator. Am J Physiol Heart Circ Physiol 283:H949-H957.

Aasum E, Cooper M, Severson DL, and Larsen TS (2005) Effect of BM 17.0744, a PPAR $\alpha$ ligand, on the metabolism of perfused hearts from control and diabetic mice. Can J Physiol Pharmacol 83:183-190.

Andreadou I, Efentakis P, Balafas E, Togliatto G, Davos CH, Varela A, Dimitriou CA Nikolaou PE, Maratou E, Lambadiari V, et al. (2017) Empagliflozin limits myocardial infarction in vivo and cell death in vitro: role of STAT3, mitochondria, and redox aspects. Front Physiol 8:1077.

Aubert G, Martin OJ, Horton JL, Lai L, Vega RB, Leone TC, Koves T, Gardell SJ, Krüger M, Hoppel CL, et al. (2016) The failing heart relies on ketone bodies as a fuel. Circulation 133:698-705.

Ayoub KF, Pothineni NVK, Rutland J, Ding Z, and Mehta JL (2017) Immunity, inflammation, and oxidative stress in heart failure: emerging molecular targets. Cardiovasc Drugs Ther 31:593-608.

Bedi KC Jr., Snyder NW, Brandimarto J, Aziz M, Mesaros C, Worth AJ, Wang LL, Javaheri A, Blair IA, Margulies KB, et al. (2016) Evidence for intramyocardial disruption of lipid metabolism and increased myocardial ketone utilization in advanced human heart failure. Circulation 133:706-716.

Bi S and Moran TH (2016) Obesity in the Otsuka Long Evans Tokushima fatty rat: mechanisms and discoveries. Front Nutr 3:21.

Buler M, Andersson U, and Hakkola J (2016) Who watches the watchmen? Regulation of the expression and activity of sirtuins. FASEB $J$ 30:3942-3960.

Carley AN, Semeniuk LM, Shimoni Y, Aasum E, Larsen TS, Berger JP, and Severson DL (2004) Treatment of type 2 diabetic $d b / d b$ mice with a novel PPAR $\gamma$ agonist improves cardiac metabolism but not contractile function. Am J Physiol Endocrinol Metab 286:E449-E455.

Cubbon RM, Adams B, Rajwani A, Mercer BN, Patel PA, Gherardi G, Gale CP, Batin PD, Ajjan R, Kearney L, et al. (2013) Diabetes mellitus is associated with adverse prognosis in chronic heart failure of ischaemic and non-ischaemic aetiology. Diab Vasc Dis Res 10:330-336.

Diakos NA, Navankasattusas S, Abel ED, Rutter J, McCreath L, Ferrin P, McKellar SH, Miller DV, Park SY, Richardson RS, et al. (2016) Evidence of glycolysis up-regulation and pyruvate mitochondrial oxidation mismatch during mechanical unloading of the failing human heart: implications for cardiac reloading and conditioning. JACC Basic Transl Sci 1:432-444.

Ferrannini E, Baldi S, Frascerra S, Astiarraga B, Heise T, Bizzotto R, Mari A, Pieber TR, and Muscelli E (2016a) Shift to fatty substrate utilization in response to sodium-glucose cotransporter 2 inhibition in subjects without diabetes and patients with type 2 diabetes. Diabetes 65:1190-1195.

Ferrannini E, Mark M, and Mayoux E (2016b) CV Protection in the EMPA-REG OUTCOME trial: a "thrifty substrate" hypothesis. Diabetes Care 39:1108-1114.

Izuta Y, Imada T, Hisamura R, Oonishi E, Nakamura S, Inagaki E, Ito M, Soga T, and Tsubota K (2018) Ketone body 3-hydroxybutyrate mimics calorie restriction via the Nrf2 activator, fumarate, in the retina. Aging Cell 17:e12699.

Kinugawa S, Tsutsui H, Hayashidani S, Ide T, Suematsu N, Satoh S, Utsumi H, and Takeshita A (2000) Treatment with dimethylthiourea prevents left ventricular remodeling and failure after experimental myocardial infarction in mice: role of oxidative stress. Circ Res 87:392-398.

Kouzu H, Miki T, Tanno M, Kuno A, Yano T, Itoh T, Sato T, Sunaga D, Murase H, Tobisawa $\mathrm{T}$, et al. (2015) Excessive degradation of adenine nucleotides by up-regulated AMP deaminase underlies afterload-induced diastolic dysfunction in the type 2 diabetic heart. J Mol Cell Cardiol 80:136-145.

Lopaschuk GD (2017) Metabolic modulators in heart disease: past, present, and future. Can J Cardiol 33:838-849.

Lopaschuk GD, Ussher JR, Folmes CD, Jaswal JS, and Stanley WC (2010) Myocardial fatty acid metabolism in health and disease. Physiol Rev 90:207-258.

Makinoshima H, Takita M, Matsumoto S, Yagishita A, Owada S, Esumi H, and Tsuchihara K (2014) Epidermal growth factor receptor (EGFR) signaling regulates global metabolic pathways in EGFR-mutated lung adenocarcinoma. $J$ Biol Chem 289:20813-20823.

Min SH, Oh TJ, Baek SI, Lee DH, Kim KM, Moon JH, Choi SH, Park KS, Jang HC, and Lim S (2018) Degree of ketonaemia and its association with insulin resistance after dapagliflozin treatment in type 2 diabetes. Diabetes Metab 44:73-76.

Mizuno M, Kuno A, Yano T, Miki T, Oshima H, Sato T, Nakata K, Kimura Y, Tanno M, and Miura T (2018) Empagliflozin normalizes the size and number of mitochondria and prevents reduction in mitochondrial size after myocardial infarction in diabetic hearts. Physiol Rep 6:e13741.

Mizuno Y, Harada E, Nakagawa H, Morikawa Y, Shono M, Kugimiya F, Yoshimura $\mathrm{M}$, and Yasue H (2017) The diabetic heart utilizes ketone bodies as an energy source. Metabolism 77:65-72.

Mudaliar S, Alloju S, and Henry RR (2016) Can a shift in fuel energetics explain the beneficial cardiorenal outcomes in the EMPA-REG OUTCOME study? a unifying hypothesis. Diabetes Care 39:1115-1122.

Münzel T, Gori T, Keaney JF Jr., Maack C, and Daiber A (2015) Pathophysiological role of oxidative stress in systolic and diastolic heart failure and its therapeutic implications. Eur Heart $J$ 36:2555-2564.

Murase H, Kuno A, Miki T, Tanno M, Yano T, Kouzu H, Ishikawa S, Tobisawa T, Ogasawara M, Nishizawa K, et al. (2015) Inhibition of DPP-4 reduces acute mortality after myocardial infarction with restoration of autophagic response in type 2 diabetic rats. Cardiovasc Diabetol 14:103.

Nagao M, Toh R, Irino Y, Mori T, Nakajima H, Hara T, Honjo T, Satomi-Kobayashi S, Shinke T, Tanaka H, et al. (2016) $\beta$-Hydroxybutyrate elevation as a compensatory response against oxidative stress in cardiomyocytes. Biochem Biophys Res Commun 475:322-328.

Neal B, Perkovic V, Mahaffey KW, de Zeeuw D, Fulcher G, Erondu N, Shaw W, Law G, Desai M, and Matthews DR; CANVAS Program Collaborative Group (2017) 
Canagliflozin and cardiovascular and renal events in type 2 diabetes. $N$ Engl J Med 377:644-657.

Nichols GA, Gullion CM, Koro CE, Ephross SA, and Brown JB (2004) The incidence of congestive heart failure in type 2 diabetes: an update. Diabetes Care 27: 1879-1884.

Niemann B, Rohrbach S, Miller MR, Newby DE, Fuster V, and Kovacic JC (2017) Oxidative stress and cardiovascular risk: obesity, diabetes, smoking, and pollution: part 3 of a 3-part series. J Am Coll Cardiol 70:230-251.

Obokata M, Negishi K, Sunaga H, Ishida H, Ito K, Ogawa T, Iso T, Ando Y, and Kurabayashi M (2017) Association between circulating ketone bodies and worse outcomes in hemodialysis patients. $J$ Am Heart Assoc 6:e00685.

Satterstrom FK, Swindell WR, Laurent G, Vyas S, Bulyk ML, and Haigis MC (2015) Nuclear respiratory factor 2 induces SIRT3 expression. Aging Cell 14:818-825.

Schugar RC, Moll AR, André d'Avignon D, Weinheimer CJ, Kovacs A, and Crawford PA (2014) Cardiomyocyte-specific deficiency of ketone body metabolism promotes accelerated pathological remodeling. Mol Metab 3:754-769.

Shah AD, Langenberg C, Rapsomaniki E, Denaxas S, Pujades-Rodriguez M, Gale CP, Deanfield J, Smeeth L, Timmis A, and Hemingway H (2015) Type 2 diabetes and incidence of cardiovascular diseases: a cohort study in 1.9 million people. Lancet Diabetes Endocrinol 3:105-113.

Shimazu T, Hirschey MD, Newman J, He W, Shirakawa K, Le Moan N, Grueter CA, Lim H, Saunders LR, Stevens RD, et al. (2013) Suppression of oxidative stress by $\beta$-hydroxybutyrate, an endogenous histone deacetylase inhibitor. Science 339:211-214.

Takada A, Miki T, Kuno A, Kouzu H, Sunaga D, Itoh T, Tanno M, Yano T, Sato T, Ishikawa S, et al. (2012) Role of ER stress in ventricular contractile dysfunction in type 2 diabetes. PLoS One 7:e39893.

Tseng AH, Wu LH, Shieh SS, and Wang DL (2014) SIRT3 interactions with FOXO3 acetylation, phosphorylation and ubiquitinylation mediate endothelial cell responses to hypoxia. Biochem J 464:157-168.
Uchihashi M, Hoshino A, Okawa Y, Ariyoshi M, Kaimoto S, Tateishi S, Ono K, Yamanaka R, Hato D, Fushimura Y, et al. (2017) Cardiac-specific Bdh1 overexpression ameliorates oxidative stress and cardiac remodeling in pressure overload-induced heart failure. Circ Heart Fail 10:e004417.

Udell JA, Cavender MA, Bhatt DL, Chatterjee S, Farkouh ME, and Scirica BM (2015) Glucose-lowering drugs or strategies and cardiovascular outcomes in patients with or at risk for type 2 diabetes: a meta-analysis of randomised controlled trials. Lancet Diabetes Endocrinol 3:356-366.

Verma S, Rawat S, Ho KL, Wagg CS, Zhang L, Teoh H, Dyck JE, Uddin GM, Oudit GY, Mayoux E, et al. (2018) Empagliflozin increases cardiac energy production in diabetes: novel translational insights into the heart failure benefits of SGLT2 inhibitors. JACC Basic Transl Sci 3:575-587.

Wentz AE, d'Avignon DA, Weber ML, Cotter DG, Doherty JM, Kerns R, Nagarajan R, Reddy N, Sambandam N, and Crawford PA (2010) Adaptation of myocardial substrate metabolism to a ketogenic nutrient environment. $J$ Biol Chem 285: 24447-24456.

Yin J, Han P, Tang Z, Liu Q, and Shi J (2015) Sirtuin 3 mediates neuroprotection of ketones against ischemic stroke. J Cereb Blood Flow Metab 35:1783-1789.

Zinman B, Wanner C, Lachin JM, Fitchett D, Bluhmki E, Hantel S, Mattheus M, Devins T, Johansen OE, Woerle HJ, et al.; EMPA-REG OUTCOME Investigators (2015) Empagliflozin, cardiovascular outcomes, and mortality in type 2 diabetes. N Engl J Med 373:2117-2128.

Address correspondence to: Dr. Tetsuji Miura, Department of Cardiovascular, Renal, and Metabolic Medicine, Sapporo Medical University School of Medicine, South-1, West-16, Chuo-ku, Sapporo 060-8543, Japan. E-mail: miura@sapmed.ac.jp 